\title{
Power spectral density of the heterogeneous fracture compliance from scattered elastic wavefields
}

\author{
Shohei Minato ${ }^{1}$ and Ranajit Ghose ${ }^{1}$
}

\begin{abstract}
Using the scattered elastic wavefield, a method to derive the power spectral density (PSD) of the heterogeneous compliance distribution, along the plane of a single fracture, is formulated. The method involves estimation of the stress field at the fracture depth from the scattered wavefield followed by least-squares optimization of the PSD of the stress field. We consider a 2D geometry and incident plane waves. The derivations are made in the frequency-wavenumber domain. To derive the relationship between the scattered wavefield and the PSD of the heterogeneous compliance, perturbation theory is used. In the forward modeling, the scattering response of the heterogeneous compliance is estimated, assuming a stationary random process. Numerical tests of the proposed method of PSD estimation offer important insights. The estimated PSD of the compliance (given by the standard deviation $\delta$ and the correlation length $l_{c}$,
\end{abstract}

assuming a Gaussian distribution) as a statistical measure of the heterogeneous fracture compliance is robust against fracture depth uncertainties. Furthermore, the sparse spatial sampling of the wavefield is not problematic as long as the data contain those wavenumber components which represent the slope of the PSD of the stress field. However, when the sampling interval is too large compared to the correlation length of the heterogeneous compliance distribution, it is difficult to estimate accurately the PSD of the compliance due to spatial aliasing and small amplitude variation within the available wavenumber. For a given spatial sampling, the use of higher frequencies results in a stronger amplitude variation in the scattered wavefield. This leads to better estimates of the PSD of the compliance via a least-squares optimization. In the presence of white noise, a change in the PSD of the stress field at the fracture depth significantly affects the PSD estimates. In this case, a data-driven amplitude correction improves the estimates of $\delta$ and $l_{c}$.

\section{INTRODUCTION}

Fracture compliance relates to the deformation at the plane of weakness (fracture plane) due to an applied stress. The linear-slip model (Schoenberg, 1980) assumes a linear relationship between the elastic-wave-induced, small relative displacement and the stress across a fracture. The validity of this model has been confirmed earlier in physical and numerical experiments (e.g., Pyrak-Nolte et al., 1990; Haugen and Schoenberg, 2000; Toomey et al., 2002).

Fracture compliance has been related to the microscale properties like the roughness of the fracture interface and the normal stress (Brown and Scholz, 1985) or the distribution of the contact area and the fluid-filled aperture (Hudson et al., 1996). These microscale properties are generally characterized in a statistical manner in rock physics (e.g., Lanaro, 2000). A reasonable statistical description of a presumably random process like the spatial heterogeneity of the microscale fracture properties is given by the power spectral density (PSD) and the probability density function (PDF), which have been used in the past to model the topography of a rough fracture surface (e.g., Brown, 1995).

Using numerical modeling, Pyrak-Nolte and Morris (2000) have investigated the relation between the random distribution of fracture aperture characterized by the correlation length, the specific fracture compliance, and the fluid flow. At large elastic wavelengths, the estimated fracture compliance reflects an average effect of the microstructures (e.g., Hudson et al., 1996). However, if the characteristics of the random distribution of fracture compliance can be extracted from the elastic wavefield, with the correlation length and the standard deviation reflecting the random distribution, then this information can be useful in characterizing the fluid flow properties.

In earlier laboratory studies, the fracture compliance has generally been estimated from the reflection or transmission coefficients

\footnotetext{
Manuscript received by the Editor 3 May 2013; revised manuscript received 8 October 2013; published online 17 February 2014.

${ }^{1}$ Delft University of Technology, Department of Geoscience and Engineering, The Netherlands. E-mail: s.minato@tudelft.nl; r.ghose@tudelft.nl.

(C) 2014 Society of Exploration Geophysicists. All rights reserved.
} 
(e.g., Pyrak-Nolte et al., 1990; Lubbe et al., 2008), assuming homogeneous compliance distribution. Pyrak-Nolte and Nolte (1992) have measured the compliance of a single fracture from the transmission coefficient and showed that the assumption of spatially heterogeneous distribution of the compliance along the fracture surface explains the experimental data better. There is no approach, so far, to directly estimate the statistical properties of the heterogeneous compliance distribution from the elastic data. In the present research, the goal is to derive the PSD of the spatially heterogeneous fracture compliance using the scattered elastic wavefield. This approach differs from Minato and Ghose (2013), where amplitude and phase information are used to derive the exact distribution of the laterally varying fracture compliance. In this paper, we show that it is possible to estimate the PSD of the laterally varying fracture compliance from the information of only the scattered wave amplitudes, through the use of perturbation theory.

The relation between the PSD of the volumetric heterogeneity and the scattered elastic wave is well studied in global seismology (e.g., Scherbaum and Sato, 1991; Leary, 1995). There are earlier works involving the relation between the PSD of a rough interface (welded contact or Dirichlet boundary) and the scattered elastic wave (e.g., Kuperman, 1975; Thorsos and Jackson, 1989). The approach that we derive here is different from these studies, as we focus on the elastic wave scattering from the surface heterogeneity at the interface of a nonwelded contact, considering the linear-slip model (LSM). We consider a homogeneous background medium, which is the case in many laboratory experiments. If the heterogeneity further away from the fracture is taken into consideration, additional steps of processing are needed to calculate correctly the stress field from the scattered wavefield. Leiderman et al. (2007) have developed a method to directly estimate the heterogeneous compliance distribution from the scattered wavefield. Their method assumes a multilayered background medium.

We apply perturbation theory to derive the relation between the scattered elastic wavefield and the PSD of the heterogeneous compliance. To accomplish this, we make the following two assumptions: (1) plane wave incidence, and (2) a single-fracture configuration. The derived results will, however, be useful for extension in the future to spherical waves and multiple fractures.

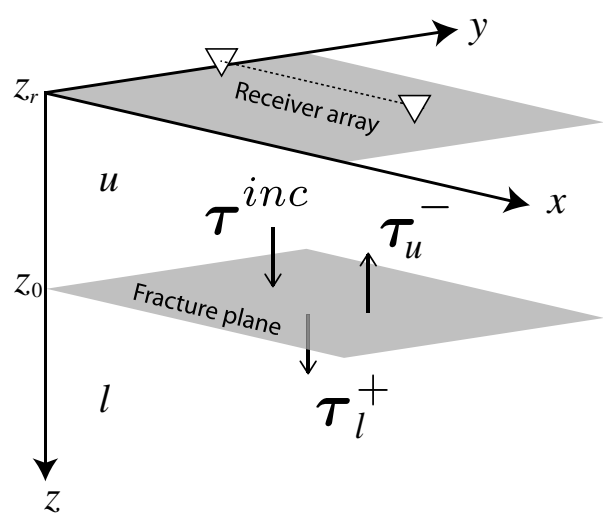

Figure 1. The configuration of scattering due to a single fracture. Receivers are assumed to be located in the $x-y$ plane at $z=z_{r}$ and the fracture is located in the $x-y$ plane at $z=z_{0} . \tau^{\text {inc }}, \boldsymbol{\tau}_{u}^{-}$, $\boldsymbol{\tau}_{l}^{+}$are the incident stress field, the upgoing stress field in the upper medium, and the downgoing stress field at the lower medium, respectively.
The structure of this paper is as follows. First, we solve the forward problem to estimate the scattered elastic wavefield due to the heterogeneous compliance of a single fracture. We then apply perturbation theory to approximate the solution and to analyze the first-order scattering. Next, we develop a method for estimating the PSD of the heterogeneous compliance from the scattered wavefield using perturbation theory. This is achieved by estimating the stress field at the fracture depth from the scattered wavefield followed by a least-square optimization of the predicted PSD of the stress field. Finally, we illustrate the new approach through numerical examples. We investigate the performance of our method considering the following factors: (1) the presence of errors in the input parameters; e.g., fracture depth, value of the background compliance, and medium velocity, (2) the effect of sparse sampling of the data in space domain, (3) the effect of the correlation length of the heterogeneous compliance, (4) the effect of frequency content, and (5) the presence of white noise.

Note that the method requires the medium parameters (density and velocity), fracture depth, and background compliance to be known. In order to apply the method to field seismic data, these values might be obtained from logging data. The fracture depth and the background compliance may be estimated from migration of the reflection seismic data and calculation of the reflection coefficients (e.g., Lubbe et al., 2008), respectively. We present here results for a horizontal fracture. However, the proposed approach should be useful also for nonhorizontal fractures. For that purpose, estimating the stress field at the known position of the fracture and then rotating the axes so that horizontal axes align along the dipping fracture plane are necessary.

\section{SCATTERED WAVEFIELD DUE TO A SPATIALLY HETEROGENEOUS COMPLIANCE: FORWARD MODELING}

We first obtain the exact solution for the scattered wavefield from the spatially heterogeneous compliance distribution in a homogeneous medium. The exact solution is then approximated by perturbation theory to obtain the relation between the PSD of the compliance and the PSD of the stress field.

\section{Exact solution for the scattered wavefield in relation to the $\mathbf{L S M}$}

We consider the horizontal fracture to be embedded in an elastic medium and the wavefield is recorded at the surface (Figure 1). Lubbe et al. (2008) have used horizontal fracture in their laboratory experiments. Nakagawa et al. (2004) and Leiderman et al. (2005) have also considered horizontal fractures in their numerical studies.

Considering the LSM, a fracture is modeled as a plane of weakness across which the stress $\boldsymbol{\tau}$ is continuous but the displacement $\mathbf{u}$ is discontinuous. The displacement-discontinuity $[\mathbf{u}]$ is linearly related to the applied stress $\tau$ as

$$
[\mathbf{u}](x, y)=\mathbf{Z}(x, y) \boldsymbol{\tau}(x, y),
$$

where $[\mathbf{u}]=\left(\left[\begin{array}{lll}{\left[u_{x}\right]} & {\left[u_{y}\right]} & {\left[u_{z}\right]}\end{array}\right)^{T}\right.$ and $\boldsymbol{\tau}=\left(\begin{array}{lll}\tau_{x z} & \tau_{y z} & \tau_{z z}\end{array}\right)^{T}$ are the displacement-discontinuity vector and the stress traction vector on the fracture plane, respectively. Square brackets [.] indicate the 
difference of the wavefield between just above and below the fracture plane. Here, we define the fracture to be at depth $z=z_{0}$ in the $x-y$ plane (Figure 1). We take a rotational symmetry for the fractures locally (Schoenberg, 1980) by using a diagonal compliance matrix $\mathbf{Z}$, defined as

$$
\mathbf{Z}(x, y)=\left(\begin{array}{ccc}
\eta_{T}(x, y) & 0 & 0 \\
0 & \eta_{T}(x, y) & 0 \\
0 & 0 & \eta_{N}(x, y)
\end{array}\right)
$$

where $\eta_{T}$ and $\eta_{N}$ are tangential and normal compliances, respectively. We assume these compliances to be functions of space $(x, y)$.

Nakagawa et al. (2004) were the first to derive the scattered wavefield using the displacement wave vector. To introduce the forward/inverse wavefield extrapolation operator and perturbation theory, we have recently derived the scattered wavefield by explicitly using the potential wave vector (Minato and Ghose, 2013). The exact transmission-reflection relationship in the frequency-wavenumber $(f-k)$ domain can be derived as

$$
\hat{\mathbf{L}}_{l}\left(\begin{array}{c}
\hat{\mathbf{D}}_{l}^{+} \\
\mathbf{0}
\end{array}\right)=\hat{\mathbf{L}}_{u}\left(\begin{array}{c}
\hat{\mathbf{D}}^{\mathrm{inc}} \\
\hat{\mathbf{D}}_{u}^{-}
\end{array}\right)+\left(\begin{array}{c}
i \omega \hat{\mathbf{Z}} * \hat{\boldsymbol{\tau}}_{u} \\
\mathbf{0}
\end{array}\right),
$$

where $\hat{\mathbf{D}}$ and $\hat{\mathbf{L}}$ indicate the potential wave vector and the composition matrix, respectively. The symbol * denotes convolution in the wavenumber domain. The subscripts $u$ and $l$ refer to the upper and lower media at the fracture plane (Figure 1). The superscripts + and - indicate downgoing and upgoing wavefields. The matrix $\hat{\mathbf{Z}}$ includes the Fourier transform of the fracture compliance distribution, Z. In equation 3, we assume a downgoing incident wave in the upper medium $\left(\hat{\mathbf{D}}_{u}^{+}=\hat{\mathbf{D}}^{\text {inc }}\right)$ and no upgoing wave in the lower medium $\left(\hat{\mathbf{D}}_{l}^{-}=\mathbf{0}\right.$; Figure 1$)$. Note that the potential wavefield $\hat{\mathbf{D}}^{ \pm}$relates to the particle velocity field $\hat{\mathbf{v}}$ and the stress field $\hat{\boldsymbol{\tau}}$ through the composition matrix $\hat{\mathbf{L}}$ as (e.g., Woodhouse, 1974; Ursin, 1983; Wapenaar and Berkhout, 1989)

$$
\left(\begin{array}{c}
\hat{\mathbf{v}} \\
\hat{\boldsymbol{\tau}}
\end{array}\right)=\left(\begin{array}{c}
\hat{\mathbf{v}}^{+}+\hat{\mathbf{v}}^{-} \\
\hat{\boldsymbol{\tau}}^{+}+\hat{\boldsymbol{\tau}}^{-}
\end{array}\right)=\hat{\mathbf{L}}\left(\begin{array}{c}
\hat{\mathbf{D}}^{+} \\
\hat{\mathbf{D}}^{-}
\end{array}\right)
$$

Because the wavefields are evaluated on the fracture plane $\left(z=z_{0}\right)$, it is possible to calculate the incident wavefield (downgoing wavefield) and its reflection response (upgoing wavefield) at a desired position by applying a forward wavefield extrapolation operator to propagate the potential wavefield. For example, the incident wavefield and the observed reflection response are represented as

$$
\begin{gathered}
\hat{\mathbf{D}}^{\text {inc }}\left(z_{0}\right)=\hat{\mathbf{W}}^{+}\left(z_{0}, z_{r}\right) \hat{\mathbf{D}}^{\text {inc }}\left(z_{r}\right), \\
\hat{\mathbf{D}}_{u}^{-}\left(z_{r}\right)=\hat{\mathbf{W}}^{-}\left(z_{r}, z_{0}\right) \hat{\mathbf{D}}_{u}^{-}\left(z_{0}\right),
\end{gathered}
$$

where $\hat{\mathbf{D}}^{\text {inc }}\left(z_{r}\right)$ and $\hat{\mathbf{D}}^{\text {inc }}\left(z_{0}\right)$ are the incident wave at the receiver depth and at the fracture depth, and $\hat{\mathbf{D}}_{u}^{-}\left(z_{r}\right)$ and $\hat{\mathbf{D}}_{u}^{-}\left(z_{0}\right)$ are the scattered wave at the receiver depth and at the fracture depth, respectively. The matrices $\hat{\mathbf{W}}^{ \pm}$represent the downgoing and upgoing forward wavefield extrapolation operators (equation A-3) including the propagation velocity in the upper medium and the distance of propagation $\left(\Delta z=\left|z_{0}-z_{r}\right|\right)$ from the fracture plane (see Appen$\operatorname{dix}$ A for details).

\section{Perturbation theory to approximate the scattered wavefield}

To obtain an approximate solution for the transmission/ reflection problem (equation 3), we use perturbation theory. The application of perturbation theory to the Dirichlet boundary condition can be found in Thorsos and Jackson (1989). The details of the application of perturbation theory to the LSM boundary condition is shown in Appendix B.

We apply perturbation theory to equation 3 by assuming

$$
\mathbf{Z}(x, y)=\mathbf{Z}_{0}+\epsilon \mathbf{Z}_{1}(x, y),
$$

where $\mathbf{Z}_{0}$ is a background compliance matrix and $\mathbf{Z}_{1}(x, y)$ is the perturbation from the background value. $\mathbf{Z}_{1}(x, y)$ is scaled so that it has the same order of magnitude as $\mathbf{Z}_{0}$ and $\epsilon$ is the magnitude of perturbation. By substituting equation 7 into equation 3 , and expanding the wavefield into a power series of $\epsilon$, we solve the transmission/reflection problem for various orders of $\epsilon$. For the zeroth order, it yields (see Appendix B for details)

$$
\hat{\mathbf{L}}_{l}\left(\begin{array}{c}
\hat{\mathbf{D}}_{l}^{+(0)} \\
\mathbf{0}
\end{array}\right)=\left(\begin{array}{cc}
\mathbf{I} & i \omega \mathbf{Z}_{0} \\
\mathbf{0} & \mathbf{I}
\end{array}\right) \hat{\mathbf{L}}_{u}\left(\begin{array}{c}
\hat{\mathbf{D}}^{\mathrm{inc}} \\
\hat{\mathbf{D}}_{u}^{-(0)}
\end{array}\right) .
$$

This equation corresponds to homogeneous (constant) compliance (e.g., Schoenberg, 1980; Haugen and Schoenberg, 2000; van Der Neut et al., 2008). For the $n$th order $(n>0)$,

$$
\hat{\mathbf{L}}_{l}\left(\begin{array}{c}
\hat{\mathbf{D}}_{l}^{+(n)} \\
\mathbf{0}
\end{array}\right)=\left(\begin{array}{cc}
\mathbf{I} & i \omega \mathbf{Z}_{0} \\
\mathbf{0} & \mathbf{I}
\end{array}\right) \hat{\mathbf{L}}_{u}\left(\begin{array}{c}
\mathbf{0} \\
\hat{\mathbf{D}}_{u}^{-(n)}
\end{array}\right)+\left(\begin{array}{c}
\hat{\mathbf{S}}^{(n)} \\
\mathbf{0}
\end{array}\right),
$$

where the source term $\hat{\mathbf{S}}^{(n)}$ is constructed from the stress field of the previous order $\hat{\boldsymbol{\tau}}_{u}^{(n-1)}$ :

$$
\hat{\mathbf{S}}^{(n)}=i \omega \hat{\mathbf{Z}}_{1} * \hat{\boldsymbol{\tau}}_{u}^{(n-1)}
$$

In this paper, we illustrate the relations for the downgoing potential field in the lower medium $\hat{\mathbf{D}}_{l}^{+(i)}$ (transmitted wave) and the upgoing potential field in the upper medium $\hat{\mathbf{D}}_{u}^{-(i)}$ (reflected wave). The total response can, thus, be calculated:

$$
\hat{\mathbf{D}}_{u}^{-}=\hat{\mathbf{D}}_{u}^{-(0)}+\epsilon \hat{\mathbf{D}}_{u}^{-(1)}+\epsilon^{2} \hat{\mathbf{D}}_{u}^{-(2)} \ldots
$$

\section{ESTIMATION OF THE PSD OF THE SPATIALLY HETEROGENEOUS COMPLIANCE FROM THE SCATTERED ELASTIC RESPONSE}

\section{Deriving the relation between the PSD of the heterogeneous compliance and the PSD of the stress field at the fracture}

By using the first-order perturbation theory derived in the previous section, we can obtain the relation between the PSD of the heterogeneous compliance and the PSD of the stress field at the fracture. We consider a plane wave incident with a constant amplitude $\hat{\boldsymbol{\tau}}^{c}$ at horizontal wavenumber $\mathbf{k}^{\text {inc }}$, i.e., $\hat{\boldsymbol{\tau}}^{\text {inc }}=\hat{\boldsymbol{\tau}}^{c} \delta\left(\mathbf{k}-\mathbf{k}^{\mathrm{inc}}\right)$, 
where we introduce the notation $\mathbf{k}=\left(k_{x}, k_{y}\right)$. By substituting the incident plane wave into equation 9 and solving for the stress field, we obtain the first-order solution as

$$
\begin{aligned}
\hat{\boldsymbol{\tau}}_{u}^{-(1)}\left(\mathbf{k} ; \mathbf{k}^{\mathrm{inc}}\right)= & \left(\hat{\mathbf{H}}-i \omega \mathbf{Z}_{0}\right)^{-1} i \omega \hat{\mathbf{Z}}_{1}^{s} \\
& \times\left(\mathbf{I}+i \omega\left(\hat{\mathbf{H}}^{\mathrm{inc}}-i \omega \mathbf{Z}_{0}\right)^{-1} \mathbf{Z}_{0}\right) \hat{\boldsymbol{\tau}}^{c},
\end{aligned}
$$

where we introduce the matrix $\hat{\mathbf{H}}$ (Minato and Ghose, 2013)

$$
\begin{gathered}
\hat{\mathbf{H}}=\hat{\mathbf{L}}_{1}^{+}\left(\hat{\mathbf{L}}_{2}^{+}\right)^{-1}-\hat{\mathbf{L}}_{1}^{-}\left(\hat{\mathbf{L}}_{2}^{-}\right)^{-1} \\
\hat{\mathbf{L}}_{u}=\hat{\mathbf{L}}_{l}=\left(\begin{array}{cc}
\hat{\mathbf{L}}_{1}^{+} & \hat{\mathbf{L}}_{1}^{-} \\
\hat{\mathbf{L}}_{2}^{+} & \hat{\mathbf{L}}_{2}^{-}
\end{array}\right) .
\end{gathered}
$$

In equation $12, \hat{\mathbf{H}}^{\text {inc }}=\hat{\mathbf{H}}\left(\mathbf{k}^{\text {inc }}\right)$ and $\hat{\mathbf{Z}}_{1}^{s}$ is a "shifted" compliance function $\hat{\mathbf{Z}}_{1}^{s}=\hat{\mathbf{Z}}_{1}\left(\mathbf{k}-\mathbf{k}^{\text {inc }}\right)$.

Calculating the PSD of the upgoing stress field of the first-order solution leads to the following linear function of PSD of the perturbed normal compliance distribution (see Appendix C for details):

$$
\begin{aligned}
\lim _{A \rightarrow \infty} E\left\langle\frac{\left|\epsilon \hat{\boldsymbol{\tau}}_{u}^{-(1)}\right|^{2}}{A}\right\rangle= & f\left(\mathbf{k} ; \omega, \mathbf{k}^{\mathrm{inc}}, V_{\mathrm{S}}, V_{\mathrm{P}}, \rho, \hat{\boldsymbol{\tau}}^{c}, \nu\right) \\
& \times P_{N}\left(\mathbf{k}-\mathbf{k}^{\mathrm{inc}}\right),
\end{aligned}
$$

where $E\langle\cdot\rangle$ is an ensemble averaging, $A$ is the total area of the compliance distribution, and $P_{N}(\mathbf{k})=\lim _{A \rightarrow \infty} E\left\langle\frac{\left|\epsilon \eta_{N}^{1} / \eta_{N}^{0}\right|^{2}}{A}\right\rangle$ is the PSD of the perturbed normal compliance distribution scaled by the background compliance. The function $f(\mathbf{k})$ is independent of the PSD of the compliance and is defined by medium parameters $\left(V_{\mathrm{P}}, V_{\mathrm{S}}, \rho\right)$, amplitude of the incident stress $\left(\hat{\boldsymbol{\tau}}^{c}\right)$, angular frequency $(\omega)$, incident wavenumber $\left(\mathbf{k}^{\text {inc }}\right)$, and the compliance ratio $(\nu=$ $\left.\eta_{T} / \eta_{N}\right)$. The function $f(\mathbf{k})$ can be calculated using equations C$4, \mathrm{C}-5$, and C-6 given in Appendix C. The PSD of the total upgoing stress field at the fracture up to the first order can then be written as

$$
\lim _{A \rightarrow \infty} E\left\langle\frac{\left|\hat{\boldsymbol{\tau}}_{u}^{-}(\mathbf{k})\right|^{2}}{A}\right\rangle=f(\mathbf{k}) P_{N}\left(\mathbf{k}-\mathbf{k}^{\mathrm{inc}}\right),
$$

where $\mathbf{k} \neq \mathbf{k}^{\text {inc }}$. We use the fact that $\hat{\boldsymbol{\tau}}_{u}^{-(0)}$ has a nonzero value only in the specular wavenumber $\left(\mathbf{k}^{\mathrm{inc}}\right)$. Therefore, the nonspecular components of the PSD of the total upgoing stress field (left hand side of equation 16) are expressed by a function of the PSD of the perturbed compliance distribution using the first-order perturbation theory.

\section{Estimating the stress field at the fracture and a least-square optimization of the predicted PSD of the stress field}

To use equation 16 to estimate the PSD of the perturbed compliance distribution, we follow two steps: (1) estimating the stress field at the fracture depth from the wavefield at the receiver depth, and (2) estimating the PSD of the compliance by a least-square optimization of the predicted PSD of the stress field at the fracture.
Step 1: Estimating the stress field at the fracture depth from the wavefield at the receiver depth

First, we need to estimate the upgoing stress field at the fracture depth $\left(\hat{\boldsymbol{\tau}}_{u}^{-}\left(z_{0}\right)\right)$ from the backscattered data at the receiver depth $\left(\hat{\boldsymbol{\tau}}_{u}^{-}\left(z_{r}\right)\right.$ or $\left.\hat{\mathbf{D}}_{u}^{-}\left(z_{r}\right)\right)$. For this purpose, we apply a matched inverse operator (e.g., Wapenaar and Berkhout, 1989; Wapenaar et al., 1989) to the scattered wavefield at the receiver depth, as an approximation of the inverse wavefield extrapolation operator (see Appen$\operatorname{dix}$ A). This requires that we consider the propagating wave and suppress the evanescent wave. Furthermore, it needs the observed vector wavefield, medium velocity, and the position of the fracture plane to be known. Making use of equations 4, 14, and A-5, the upgoing stress field at the fracture depth is estimated as

$$
\hat{\boldsymbol{\tau}}_{u}^{-}\left(z_{0}\right)=\hat{\mathbf{L}}_{2}^{-} \hat{\mathbf{F}}^{-}\left(z_{0}, z_{r}\right) \hat{\mathbf{D}}_{u}^{-}\left(z_{r}\right),
$$

where $\hat{\mathbf{F}}^{-}$is the matched inverse operator (see equation A-6). Similarly, we estimate the incident stress field at the fracture depth using forward wavefield extrapolation operator (equation 5) assuming that we record the incident wavefield at the receiver depth. Therefore, the incident stress field at the fracture depth can be estimated as

$$
\hat{\boldsymbol{\tau}}^{\text {inc }}\left(z_{0}\right)=\hat{\mathbf{L}}_{2}^{+} \hat{\mathbf{W}}^{+}\left(z_{0}, z_{r}\right) \hat{\mathbf{D}}^{\text {inc }}\left(z_{r}\right)
$$

Step 2: Estimating the PSD of the compliance by a least-square optimization of the predicted PSD of the stress field

Once the stress field at the fracture is estimated, we next obtain the PSD of the perturbed compliance distribution. In this section, and in the following numerical example, we assume a 1D Gaussian distribution for the PSD of the compliance (e.g., Shapiro and $\mathrm{Hu}-$ bral, 1999)

$$
P_{N}\left(k_{x}\right)=\delta^{2} \sqrt{\pi} l_{c} e^{-k_{x}^{2} l_{c}^{2} / 4},
$$

with the corresponding autocorrelation function

$$
\gamma_{N}(x)=\delta^{2} e^{-x^{2} / l_{c}^{2}}
$$

where $\delta$ and $l_{c}$ are, respectively, the standard deviation (with respect to the background value) and the correlation length of the spatially varying random compliance distribution.

The challenge is to estimate the correct values for $\delta$ and $l_{c}$. We define our data for PSD estimation as a PSD of the stress field (left hand side of equation 16) and estimate it using a direct FFT method (e.g., Kay and Marple, 1981),

$$
d\left(k_{x}\right)=\left|\hat{\boldsymbol{\tau}}_{u}^{-}\right|^{2} / X
$$

where $X$ is the maximum fracture length and the stress field $\hat{\boldsymbol{\tau}}_{u}^{-}$is evaluated at the fracture depth after using equation 17 . Hereafter, we call this form of data as the PSD of the stress field at the fracture depth.

Equation 16 states that one can predict the PSD of the stress field at the fracture depth by substituting an arbitrary PSD function $P_{N}$. Therefore, we search for the $\delta$ and $l_{c}$ values which optimize the 
predicted PSD of the stress field. To achieve this, we minimize the energy function defined as

$$
E\left(\delta, l_{c}\right)=\sum_{k_{x}}\left|d\left(k_{x}\right)-d^{\mathrm{pred}}\left(k_{x}\right)\right|^{2},
$$

where $d^{\text {pred }}$ is the PSD of the stress field predicted from $f\left(k_{x}\right)$ and $P_{N}\left(k_{x}\right)$ in equation 16 , assuming a Gaussian form for the $P_{N}\left(k_{x}\right)$ and varying $\delta$ and $l_{c}$. The summation in equation 16 is taken along the nonspecular wavenumber component. To minimize $E\left(\delta, l_{c}\right)$, we use a grid search algorithm.

Note that the method requires the medium parameters (density and velocity), fracture depth, and background compliance to be known. These values can be measured in the laboratory experiment. When one applies the method to field seismic data, the medium parameters ought to be obtained from the logging data. The fracture depth and the background compliance may be estimated from migration of the reflection seismic data and calculation of the reflection coefficients (e.g., Lubbe et al., 2008), respectively. Further correction for background velocity and fracture depth can be done in a data-driven manner through minimization of the energy function defined by the estimated fracture compliance (Minato and Ghose, 2013). Although we present here results for horizontal fracture, the proposed approach should be useful also for nonhorizontal fractures. For that purpose, estimating the stress field at the known position of the fracture and then rotating the axes so that horizontal axes align along the dipping fracture plane are necessary. We finally assume the compliance ratios $\nu=\eta_{T} / \eta_{N}$ to be known. When applied to experimental data, it is possible to get the value of $\nu$ from $\mathrm{P}$ and $\mathrm{S}$ reflection coefficients (Lubbe et al., 2008). the experiment of Lubbe et al. (2008). We assume $\nu=1$ (i.e., $\left.\eta_{T}=\eta_{N}\right)$, which is often used to approximate a gas-filled open fracture (e.g., Sayers, 2002; Willis et al., 2006). We use the frequency range $500 \mathrm{~Hz}-2.5 \mathrm{MHz}$. The scattered response is generated by convolving the impulse response calculated by wdSDD with a Ricker wavelet of central frequency $600 \mathrm{kHz}$. Because the compliance distribution is homogeneous, the waveforms in Figure 2a are independent of the receiver position. Next, we create a heterogeneous compliance distribution model with $\delta=10 \%$ from the mean $\left(\eta_{N}^{0}=1 \times 10^{-14} \mathrm{~m} / \mathrm{Pa}\right)$, adopting the approach in the Fourier domain (Pardo-Iguzquiza and Chica-Olmo, 1993). The value $\delta=10 \%$ is inspired by the experimental data of Lanaro (2000), where the roughness of the fracture surface and its standard deviation are measured at different scale lengths. We assume that the roughness relates directly to the compliance (Brown and Scholz, 1985) and that the spatial change of the roughness $(10 \%)$ in our wavelength scale $(\approx 1 \mathrm{~cm})$ results in a $10 \%$ change of the compliance distribution around the average value. We consider three different correlation lengths $\left(l_{c}=2,4\right.$, and $\left.8 \mathrm{~cm}\right)$ and the total length of the fracture to be $2 \mathrm{~m}$. The normal compliance distributions $\left(\eta_{N}(x)=\eta_{N}^{0}+\right.$ $\epsilon \eta_{N}^{1}$ ) are modeled (Figure 2b) by using the Gaussian PSD of the perturbed compliance $\left(P_{N}\left(k_{x}\right)\right)$ shown in Figure 2c. One can see that the heterogeneous compliance distribution with shorter correlation length shows a more rapid oscillation.

Next, we compare the scattered response in time domain between spatially homogeneous and spatially heterogeneous fracture compliances. Figure $2 \mathrm{~d}$ shows the scattered wavefield when the compliance is spatially heterogeneous with $l_{c}=4 \mathrm{~cm}$ and the heterogeneity is the same as that illustrated by the black solid line in Figure 2b. In Figure 2d, the scattered response for the

\section{NUMERICAL TESTS AND DISCUSSION}

\section{Model and synthetic data}

We test 1D application (i.e., heterogeneous compliance is a function of $x$ ) of this new method using numerical examples. The geometry is 2D ( $x-z$ plane, see Figure 1). The 1D wavenumber is given by $\mathbf{k}=k_{x}$. The heterogeneous compliance distribution is modeled considering a background compliance $\left(\mathbf{Z}_{0}\right.$ or $\eta_{T}^{0}$ and $\left.\eta_{N}^{0}\right)$ and the PSD of the perturbed compliance distribution $\left(\epsilon \mathbf{Z}_{1}\right.$ or $\epsilon \eta_{T}^{1}$ and $\left.\epsilon \eta_{N}^{1}\right)$, assuming a stationary random process. We use a Gaussian PSD (equation 19) with standard deviation $\delta$ and correlation length $l_{c}$.

Nakagawa et al. (2004) have solved an equation equivalent to equation 3 , but in terms of the stress field, by discretizing the wavenumber components, which they called the wavenumberdomain seismic displacement discontinuity (wdSDD) method. Figure 2a illustrates the calculated scattered response in time domain using the wdSDD approach for a spatially homogeneous fracture $\left(\eta_{N}^{0}=1 \times 10^{-14} \mathrm{~m} / \mathrm{Pa}\right)$ of $2 \mathrm{~m}$ length embedded in a medium with $V_{\mathrm{P}}=6.3 \mathrm{~km} / \mathrm{s}$, $V_{\mathrm{S}}=3.4 \mathrm{~km} / \mathrm{s}$. The value of the compliance and the medium parameters are borrowed from a)

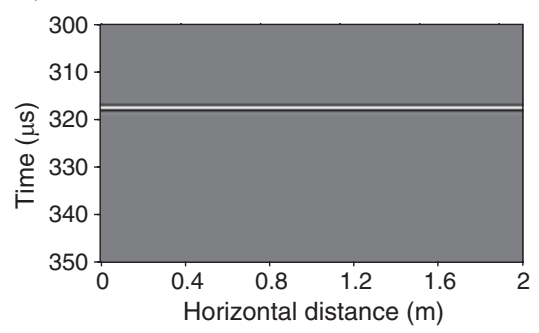

c)

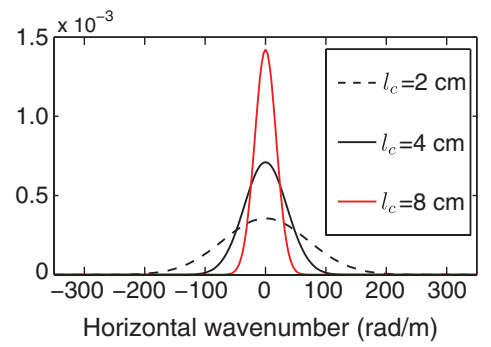

b)

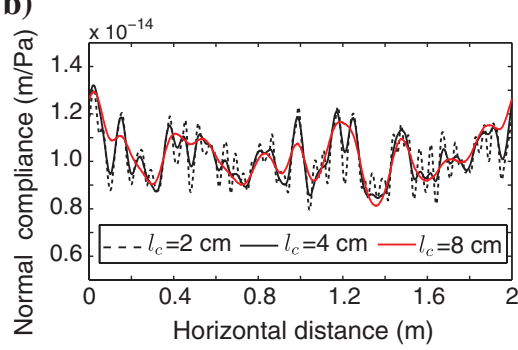

d)

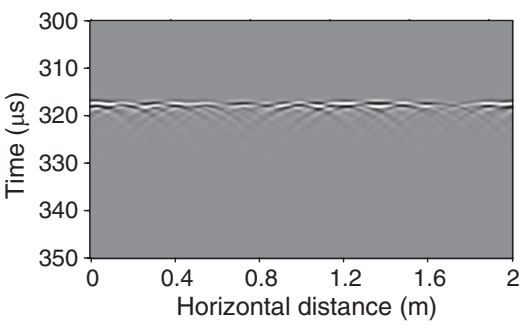

Figure 2. (a) Scattered P-wave in time domain for a homogeneous fracture, with plane $\mathrm{P}$-wave incidence. The receiver spacing is $1.25 \mathrm{~cm}$ and the fracture length is $2 \mathrm{~m}$. (b) $1 \mathrm{D}$ heterogeneous normal compliance $\eta_{N}(x)$ simulated from the Gaussian PSD shown in (c). Three different lines correspond to three different correlation lengths $\left(l_{c}=2,4\right.$, and $8 \mathrm{~cm}$ ). (c) Input Gaussian PSD of the compliance scaled relative to the background value. (d) Scattered P-wave in the time domain when the fracture compliance has spatial heterogeneity as shown by the black solid line $\left(l_{c}=4 \mathrm{~cm}\right)$ in (b). In (d), the scattered response due to a homogeneous fracture compliance (shown in [a]) is already subtracted. 
homogeneous fracture compliance (Figure 2a) is already subtracted. Other than the difference in spatial distribution of fracture compliance, all other input parameters are identical between Figures 2a and $2 d$. It is clear in Figure $2 d$ that the fine-scale scattered energy is conspicuously present in the data when the fracture compliance is spatially heterogeneous.

Next, we calculate the reflection response using the same wdSDD approach and compare the result with that using perturbation theory (equations 8-10). We consider $f=600 \mathrm{kHz}$ and use the compliance model of $l_{c}=4 \mathrm{~cm}$ (black solid line in Figure 2b). The wavenumber domain is discretized using 601 wavenumbers in the range

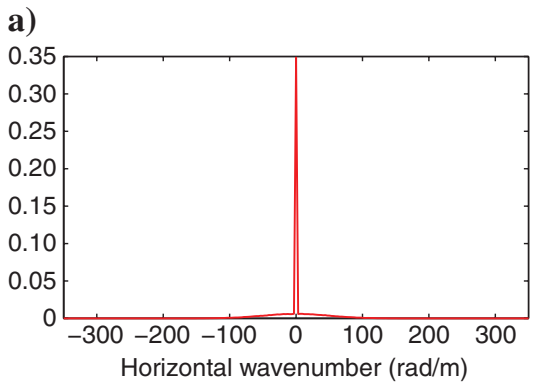

b)

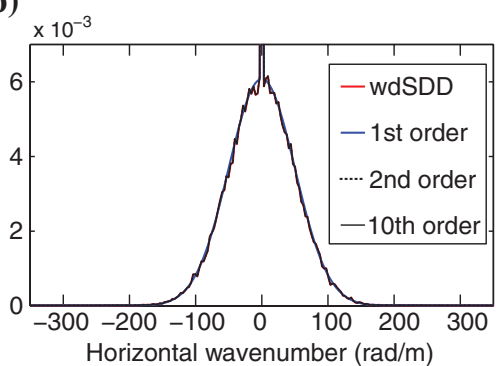

e)

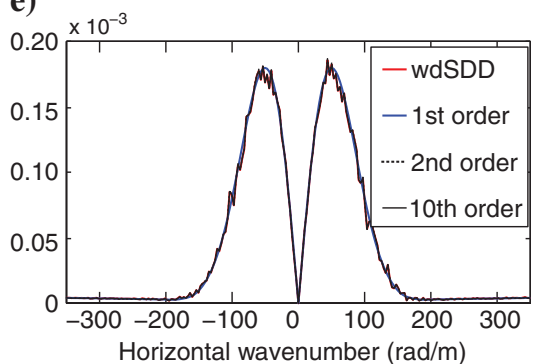

c)

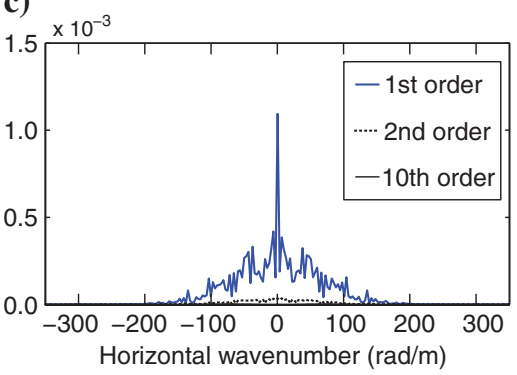

f)

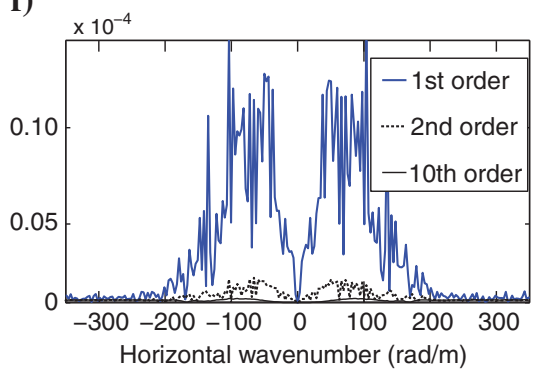

d)

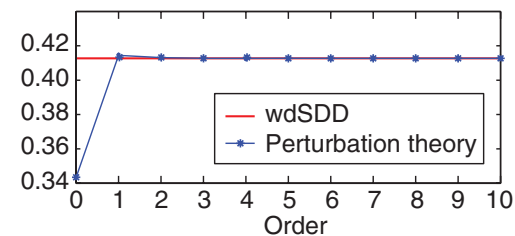

g)

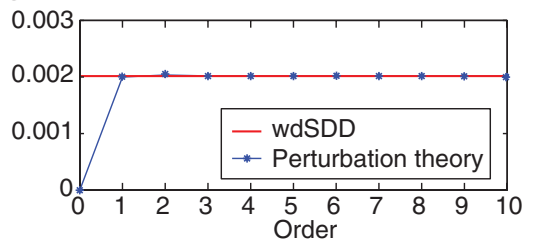

Figure 3. (a) Calculated scattered response for $\mathrm{P}$-wave amplitude in the $f-k$ domain using the wdSDD method (Nakagawa et al., 2004). (b) Enlarged view of (a) in comparison with results from perturbation theory up to the tenth order. (c) Amplitude difference between perturbation theory and the wdSDD method. (d) Amplitude of the scattered response at horizontal distance $x=0$ after inverse Fourier transformation of (b). parts (e-g) are same as (b-d), respectively, but for the SV-wave component.
$[-942,942] \mathrm{rad} / \mathrm{m}$, which corresponds to a spatial sampling interval of $0.0033 \mathrm{~m}$. For an incident P-wave, we calculate the scattered response $1 \mathrm{~m}$ above the fracture plane. Figure $3 \mathrm{a}$ shows the modeled reflection response (P-to-P reflection) at different wavenumbers for the normal incidence $\left(k_{x}^{\text {inc }}=0\right)$, using the wdSDD to the heterogeneous compliance distribution. Figure $3 b$ shows a vertically exaggerated view of Figure $3 \mathrm{a}$ for the nonspecular wavenumber components as compared with perturbation theory up to the tenth order. Figure $3 \mathrm{c}$ shows the amplitude error in case of using perturbation theory compared with the wdSDD method. Figure $3 \mathrm{~d}$ shows the amplitude at horizontal distance $x=$ 0 in the $f-x$ domain obtained by inverse Fourier transformation of the result in Figure $3 b$. From Figure $3 b-3 d$, it is clear that already after the second order, the perturbation solution approaches the wdSDD solution. Figure $3 \mathrm{e}-3 \mathrm{~g}$ are the same as Figure 3b-3d, respectively, but for the scattered SV-wave component. Due to the normal incident P-wave, the amplitude of the SV-wave at the specular wavenumber $\left(k_{x}=0\right)$ is zero. Furthermore, the amplitude of the scattered SV-wave is two orders of magnitude smaller than the P-wave amplitude (compare with Figure $3 b$ ). Because we calculate the incident $\mathrm{P}$-wave, there is no $\mathrm{SH}$-wave component. Note that the modeling of responses in the $f-k$ domain assume a periodicity of the fracture compliance distribution and that of the wavefield (incident and scattered waves) in the space domain.

Finally, we construct the synthetic data used for the PSD estimation by resampling in the space domain. To achieve this, we perform inverse Fourier transformation of the modeled data using the wdSDD method (Figure 3a) and resample the data in the space domain. We then again apply Fourier transformation to the data and obtain the pseudo-observed data in the wavenumber domain. Figure 4 shows this data (P-wave scattered response at the receiver depth) in the wavenumber domain using two different resampling intervals $(\Delta x=0.01 \mathrm{~m}$ and $\Delta x=0.05 \mathrm{~m})$ for a maximum fracture length of $2 \mathrm{~m}$. The number of samples here is 201 for $\Delta x=0.01 \mathrm{~m}$ and 41 for $\Delta x=0.05 \mathrm{~m}$. From the sampling theorem, the maximum wavenumber $\left(k_{x}^{\max }=\pi / \Delta x\right)$ is 314 for $\Delta x=0.01 \mathrm{~m}$ and 63 for $\Delta x=$ $0.05 \mathrm{~m}$. We decrease the spatial sampling to $\Delta x=0.01 \mathrm{~m}$ and $\Delta x=0.05 \mathrm{~m}$ from the finely sampled data calculated by the wdSDD method $(\Delta x=0.0033 \mathrm{~m}$; Figure 3). Because of spatial aliasing, we see small differences in the amplitude between these two data sets (blue and red line in Figure 4) and between Figure $3 b$ and Figure 4. 


\section{Estimation of PSD of the compliance on synthetic data}

We use equation 16 to estimate the PSD of the perturbed normal compliance distribution $P_{N}$ in terms of the standard deviation $\delta$ and the correlation length $l_{c}$. Note that the PSD of the perturbed tangential compliance $\left(P_{T}\right.$, where we assume $\left.\eta_{T}=\nu \eta_{N}\right)$ is sensed by the $\mathrm{SV}$-wave component. However, our data is dominated by $\mathrm{P}$-wave, as explained in the previous section. Therefore, for our data, the choice of the compliance ratio $\nu, \mathrm{S}$-wave velocity $V_{\mathrm{S}}$, and the background tangential compliance $\eta_{T}^{0}$ are insensitive to the estimated PSD $P_{N}$.

We first show the result assuming the input parameters to be accurately known. These parameters include the fracture depth $z_{0}$ and the medium velocity $V_{\mathrm{P}}$ - to estimate the stress field at the fracture depth from that at the receiver depth (step 1), and the background compliance $\eta_{N}^{0}$ and medium parameters $V_{\mathrm{P}}, \rho$ for the PSD estimation (step 2).

Figure 5a shows the estimated PSD of the stress field at the fracture depth (equation 21) using the inverse wavefield extrapolation operator (equation 17). Here, only the nonspecular components are used. We have used the data with spatial resampling interval of $\Delta x=0.01 \mathrm{~m}$. We have performed a least-square optimization of the predicted PSD of the stress field using a grid search algorithm to estimate $\delta$ and $l_{c}$ from the global minimum of the energy function $\left(E\left(\delta, l_{c}\right)\right.$ in equation 22) in the range of $0.01 \leq l_{c} \leq 0.1$ $\left(\Delta l_{c}=0.001 \mathrm{~m}\right)$ and $0.01 \leq \delta \leq 0.2(\Delta \delta=0.001 \mathrm{~m})$. Figure $5 \mathrm{~b}$ shows the energy function $E\left(\delta, l_{c}\right)$ for various values of $\delta$ and $l_{c}$. The white dot in Figure $5 \mathrm{~b}$ marks the $\delta$ and $l_{c}$ that give the global minimum $\left(\delta=0.100\right.$ and $l_{c}=0.041 \mathrm{~m}$, see Table 1$)$. The red line in Figure 5a shows the predicted PSD of the stress field derived by substituting in equation 16 the estimated values of $\delta$ and $l_{c}$ from global minimization. The values of $\delta$ and $l_{c}$ are accurately estimated by the least-square optimization.

We have illustrated these results when the input parameters are assumed to be known accurately and the data is noise-free and have sufficient sampling in the $f-k$ domain. In real applications, however, these assumptions are hardly met. Therefore, we investigate and discuss the performance of this new method with regard to the following five issues: (1) the presence of error in the input parameters, (2) the effect of sparse sampling of data in the space domain, (3) the effect of the correlation length of the heterogeneous compliance, (4) the effect of frequency, and (5) the presence of white noise. the wavefield. However, the fracture depth with two different values $\left(z_{0}^{\text {est }}=z_{0}+\lambda / 10\right.$ and $\left.z_{0}^{\text {est }}=0\right)$ did not show any difference in $\delta$ and $l_{c}$ estimated by least-square optimization. To check this effect, we show in Figure 6a the data by substituting $z_{0}^{\text {est }}=0$ (without phasecorrection due to the wavefield extrapolation operator) and $z_{0}^{\text {est }}=z_{0}$ (true phase correction due to the wavefield extrapolation operator). Note that these two data are almost the same. Further, we have calculated the data substituting different values of $z_{0}^{e s t}$ and computed the average difference from the true value as

$$
\operatorname{Error}\left(z_{0}^{\text {est }}\right)=\sqrt{\frac{\sum_{k_{x}}\left(d^{\text {true }}\left(k_{x}\right)-d\left(k_{x} ; z_{0}^{\text {est }}\right)\right)^{2}}{N}},
$$

where $d^{\text {true }}\left(k_{x}\right)$ is obtained after applying the true phase correction. We find that the value of the error oscillates (Figure 6b). However, the amplitude of the error is much $\left(\approx 10^{-4}\right.$ times $)$ smaller than data amplitude (Figure 5a). This result suggests that the estimated PSD is robust against the fracture depth uncertainties, shown in Table 1.

Here, we have assumed the background medium to be homogeneous in the vicinity of the fracture. The background can be heterogeneous further away. But in that case, one needs to take additional care to estimate correctly the stress field at the fracture from the scattering data.

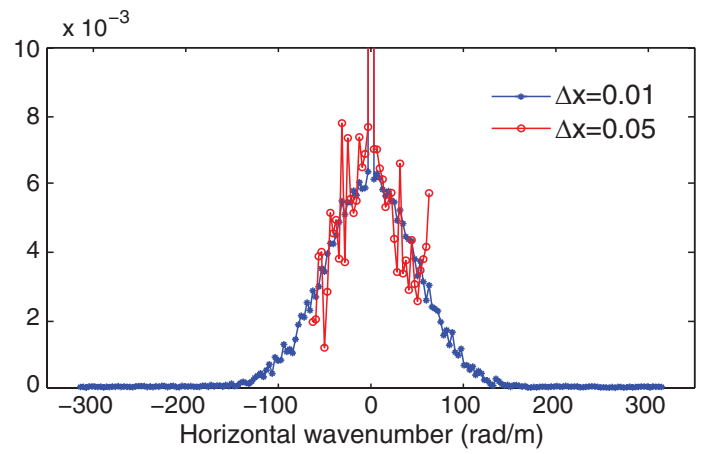

Figure 4. Amplitude of synthetic P-wave data resampled in space domain, with sampling intervals $\Delta x=0.01 \mathrm{~m}$ (blue line) and $\Delta x=$ $0.05 \mathrm{~m}$ (red line).

\section{The effect of the errors in the input parameters}

We examine the effect of errors in the input value of fracture depth $z_{0}^{\text {est }}$, background compliance $\eta_{N}^{0 \text { (est) }}$ and medium velocity $V_{\mathrm{P}}^{\text {est }}$ in the PSD estimation. Table 1 summarizes the estimated parameters of PSD of the compliance (value of $\delta$ and $l_{c}$ ) for different values of $z_{0}^{\text {est }}, \eta_{N}^{\text {(est) }}$, and $V_{\mathrm{P}}^{\text {est }}$. One can see that the errors in $\eta_{N}^{O \text { (est) }}$ and $V_{\mathrm{P}}^{\text {est }}$ have a larger effect on the estimated $\delta$ than on the estimated $l_{c}$. The fracture depth and the medium velocity are used to estimate the stress field at the fracture by forward/inverse wavefield extrapolation operators (equations 17 and 18). This can affect the estimation of the phase of a)

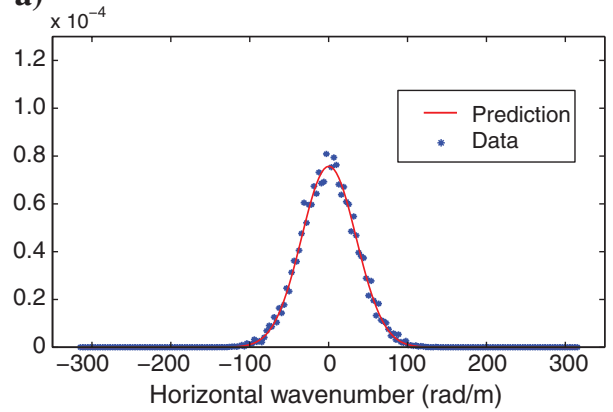

b)

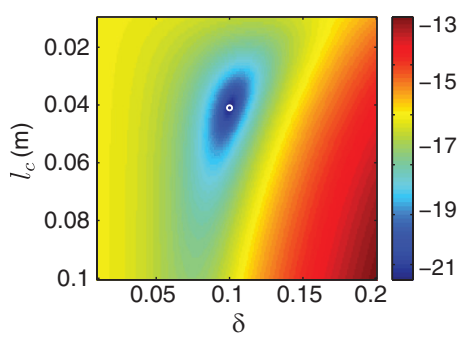

Figure 5. (a) PSD of stress field at the fracture depth, with $\Delta x=0.01 \mathrm{~m}$ and prediction using $\delta$ and $l_{c}$ estimated by grid search optimization of the energy function. (b) Energy function for grid search in the $\log$ scale $\left(\log _{10} E\left(\delta, l_{c}\right)\right)$. 


\section{The effect of spatial sampling}

Spatial sampling interval controls the available maximum wavenumber in the data. We examine the effect of undersampling the data in the space domain. Blue asterisks in Figure 7a show the PSD of the stress field at the fracture depth estimated from the sparsely sampled data $(\Delta x=0.05 \mathrm{~m})$. The red line in Figure $7 \mathrm{a}$ shows the PSD of the stress field estimated by least-square optimization for the model with $l_{c}=4 \mathrm{~cm}$. The true PSD (black line in Figure $7 \mathrm{a}$ ) is derived from equation 16 . Figure $7 \mathrm{~b}$ shows the energy function of the least-square optimization. The estimated values $\left(\delta=0.103\right.$ and $\left.l_{c}=0.045 \mathrm{~m}\right)$ are not very accurate. Due to a lack of the higher wavenumber components in the data for $\Delta x=0.05 \mathrm{~m}$ $\left(k_{x}^{\max } \approx 63\right)$, which give the characteristics of the slope of the PSD of the stress field, the estimation of $l_{c}$ is slightly inferior to that when $\Delta x=0.01 \mathrm{~m}\left(k_{x}^{\max } \approx 314\right)$, see Figure 5. Note that, in the sparsely sampled data $(\Delta x=0.05 \mathrm{~m})$, the higher wavenumbers $\left(k_{x} \geq 63\right)$ are superimposed on the lower wavenumbers due to aliasing. It affects the estimation of the PSD of the stress field at the fracture depth (compare the blue asterisks between Figure 5a and Figure 7a). This is also the reason why the result is inferior to that with $\Delta x=0.01 \mathrm{~m}$. It is, however, clear that the estimated PSD of the stress field (Figure 7a) preserves the information of $\delta$ and $l_{c}$. This is because the amplitudes at higher wavenumbers are smaller than those at lower wavenumbers due to the normal incidence in case of synthetic data with $l_{c}=4 \mathrm{~cm}$.

\section{The effect of the correlation length}

In the previous subsection, we have used synthetic data with $l_{c}=4 \mathrm{~cm}$. In this subsection, we use synthetic data with different correlation lengths $l_{c}=2$ and $8 \mathrm{~cm}$ (Figure 2b) to examine the effect on the PSD estimation. As in the previous section, here also we examine the estimated PSD of the stress field at the fracture depth for sampling interval $\Delta=0.05 \mathrm{~m}$ and the result of the least-square optimization for data with $l_{c}=2 \mathrm{~cm}$ (Figure 7c and 7d) and $l_{c}=8 \mathrm{~cm}$ (Figure 7e and 7f). The estimated values of $\epsilon$ and $l_{c}$ are summarized in Table 2 and 3.

Clearly, the data with $l_{c}=2 \mathrm{~cm}$ is more scattered (Figure 7c) than those with longer correlation lengths (Figure 7a and 7e). This is because the compliance model with short correlation length contains larger amplitudes at higher wavenumbers than that with long correlation length (Figure 2c). Therefore, the data is significantly affected by spatial aliasing (sparse sampling). Furthermore, the true PSD of the stress field with $l_{c}=2 \mathrm{~cm}$ calculated from equation 16 (black line in Figure 7c) shows small amplitude variation in the available range of the wavenumber (compare the black lines between Figure $7 \mathrm{c}$ and $7 \mathrm{e}$ ). These observations suggest that when the correlation length of the heterogeneous compliance distribution is small compared to the sampling interval, it is difficult to estimate the PSD of the stress field accurately. The energy functions (Figure $7 \mathrm{~b}, 7 \mathrm{~d}$, and 7f) show that the data with longer correlation length have larger gradient around the minimum. This indicates that the approach is more robust if the correlation length is large, for a given spatial sampling.

In our examples, the data with correlation lengths close to the sampling interval $\left(l_{c}=4 \mathrm{~cm}\right.$ and $\left.\Delta x=5 \mathrm{~cm}\right)$ provide relatively ac- a)

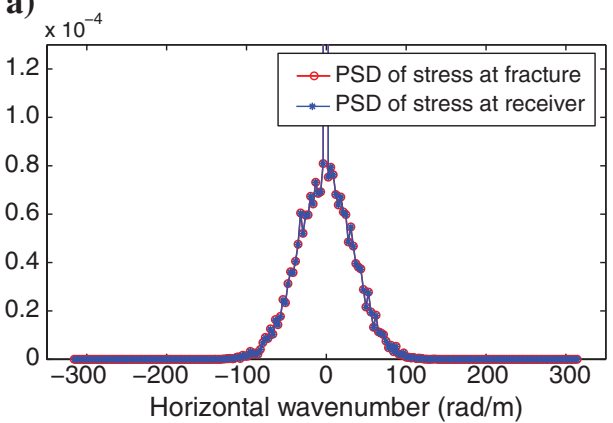

b)

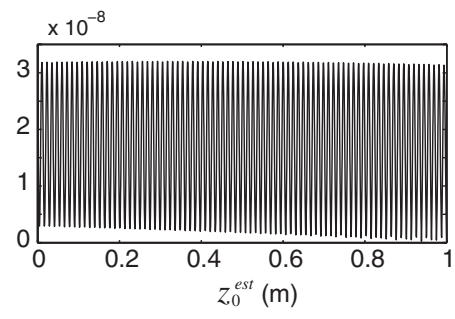

Figure 6. (a) PSD of stress field calculated at the fracture depth (red line) and at the receiver depth (blue line). (b) Average error in the PSD of stress field for different values of the fracture depth $z_{0}^{\text {est }}$.

Table 1. Summary of the estimated $\delta$ and $l_{c}$ by least-square optimization, with various values for the input parameters.

\begin{tabular}{|c|c|c|c|c|c|c|}
\hline$\Delta x(\mathrm{~m})$ & $z_{0}^{\text {est }}$ & $\eta_{N}^{0 \text { (est) }}$ & $V_{\mathrm{P}}^{\mathrm{est}}$ & $\mathrm{S} / \mathrm{N}$ & $\delta$ & $l_{c}(\mathrm{~m})$ \\
\hline True model & $z_{0}$ & $\eta_{N}^{0}$ & $V_{\mathrm{P}}$ & $\infty$ & 0.100 & 0.040 \\
\hline 0.05 & $z_{0}$ & $\eta_{N}^{0}$ & $V_{\mathrm{P}}$ & $\infty$ & $0.103 \pm 0.001$ & $0.045 \pm 0.001$ \\
\hline 0.01 & $z_{0}+\lambda / 10$ & $\eta_{N}^{0}$ & $V_{\mathrm{P}}$ & $\infty$ & $0.100 \pm 0.001$ & $0.041 \pm 0.001$ \\
\hline 0.01 & $z_{0}$ & $0.5 \eta_{N}^{0}$ & $V_{\mathrm{P}}$ & $\infty$ & $0.183 \pm 0.001$ & $0.041 \pm 0.001$ \\
\hline 0.01 & $z_{0}$ & $\eta_{N}^{0}$ & $1.05 V_{\mathrm{P}}$ & $\infty$ & $0.096 \pm 0.001$ & $0.041 \pm 0.001$ \\
\hline 0.01 & $z_{0}$ & $\eta_{N}^{0}$ & $0.95 V_{\mathrm{P}}$ & $\infty$ & $0.104 \pm 0.001$ & $0.041 \pm 0.001$ \\
\hline 0.01 & $z_{0}$ & $\eta_{N}^{0}$ & $V_{\mathrm{P}}$ & 700 & $0.101 \pm 0.001$ & $0.041 \pm 0.001$ \\
\hline
\end{tabular}


curate estimates of $\epsilon$ and $l_{c}$ : The deviation of the estimated $l_{c}$ from the true value is about $10 \%$ (see Table 1). However, when the correlation length decreases to about half of the sampling interval $\left(l_{c}=2 \mathrm{~cm}\right.$ and $\left.\Delta x=5 \mathrm{~cm}\right), \epsilon$ and $l_{c}$ are not accurately estimated: the deviation of the estimated $l_{c}$ from the true value is then about $40 \%$ (see Table 2). We therefore conclude that it is a combination of correlation length and spatial sampling that determines the accuracy of the predicted PSD of the laterally varying fracture compliance. amplitude shift especially at higher wavenumbers. This is because we assume the white noise to have a constant amplitude spectrum and the higher wavenumber components to be dominated by noise. The change in the shape of the PSD of the stress field at the fracture clearly affects the estimation of $\delta$ and $l_{c}$. We have found that these errors can be effectively corrected by subtracting a constant amplitude from the data. This has been performed in a data-driven manner. Red line in Figure 9 shows the PSD of the stress field estimated

\section{The effect of frequency}

As shown earlier, the slope of the PSD of the stress field is important for high-quality estimation. The PSD of the stress field at the fracture depth can be frequency dependent. Therefore, we examine the effect of frequency on the PSD estimation.

Figure 8 shows the PSD of the stress field at the fracture depth calculated using wdSDD with fixed values for the medium parameters and the PSD of the compliance $\left(l_{c}=4 \mathrm{~cm}\right)$. Here we use three different incident frequencies: 100, 300, and $600 \mathrm{kHz}$, assuming that the PSD of the compliance does not change with frequency. Evidently, a higher frequency corresponds to a higher amplitude variation in the calculated PSD of the stress field. This indicates that the higher frequencies in the data produce stronger scattering than the lower frequencies. A strong amplitude variation leads to a high sensitivity in the least-square optimization. The use of high frequencies will result in a better estimation of the PSD of the compliance.

\section{The effect of white noise}

Finally, we look at the impact of the presence of white noise in the data on the PSD estimation. We introduce white noise in data in the $f-k$ domain assuming a constant amplitude spectrum but random phase fluctuations. The amplitude of the noise is determined by the signal-to-noise ratio $(\mathrm{S} / \mathrm{N})$ defined by the value relative to the maximum amplitude in the noise-free data. We use data with $\Delta x=0.01 \mathrm{~m}$ (Figure 4), the $\mathrm{S} / \mathrm{N}$ is set to 700 and 150 . The results are presented in Table 1. Note that the estimated values of $\delta$ and $l_{c}$ using $\mathrm{S} / \mathrm{N}=700$ (estimated values being $\left.\delta=0.101 \pm 0.001, l_{c}=0.041 \pm 0.001 \mathrm{~m}\right)$ are almost same as the true values $\left(\delta=0.1, l_{c}=\right.$ $0.04 \mathrm{~m})$. However, when $\mathrm{S} / \mathrm{N}=150$, the estimated values $\left(\delta=0.213 \pm 0.001, l_{c}=0.007\right.$ $\pm 0.001 \mathrm{~m}$ ) show a significant deviation from the true values. Figure 9 shows data with $\mathrm{S} / \mathrm{N}$ $=150$ and the predicted PSD of the stress field from the least-square optimization (black dashed line). It is clear that these data have larger amplitudes than the original data (Figure 5a) due to the contamination with white noise. Furthermore, the increase in amplitude shows up as a constant
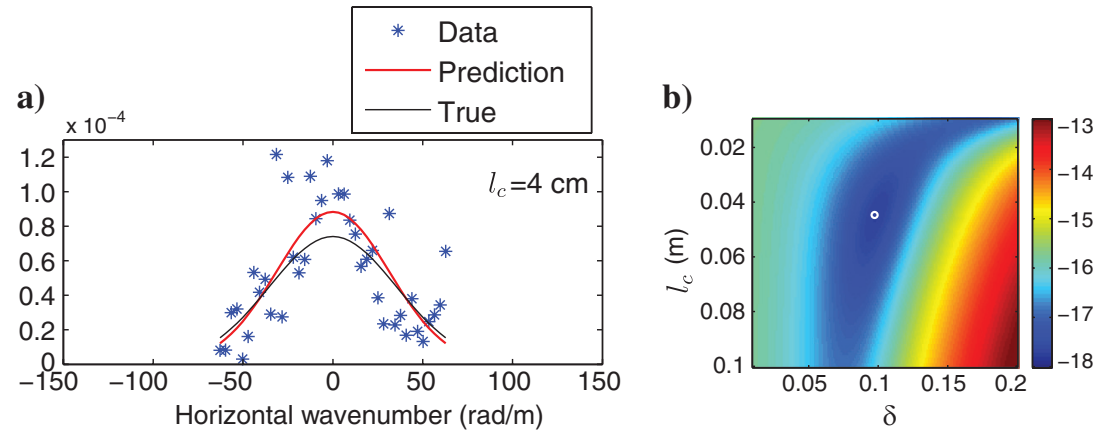

c)

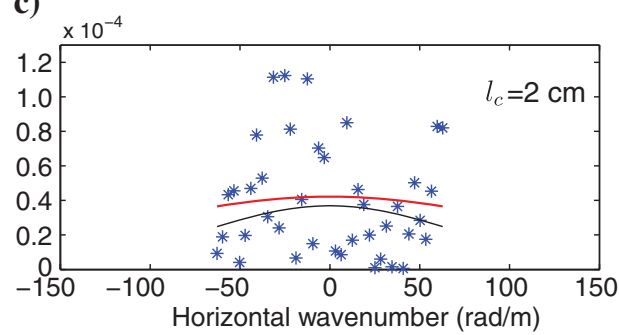

d)

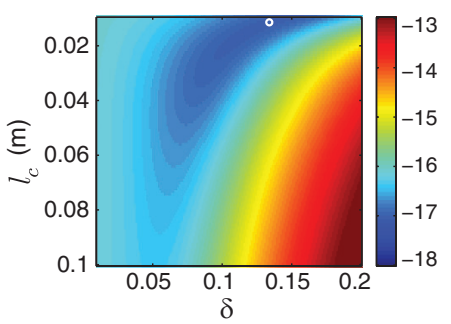

f)

e)
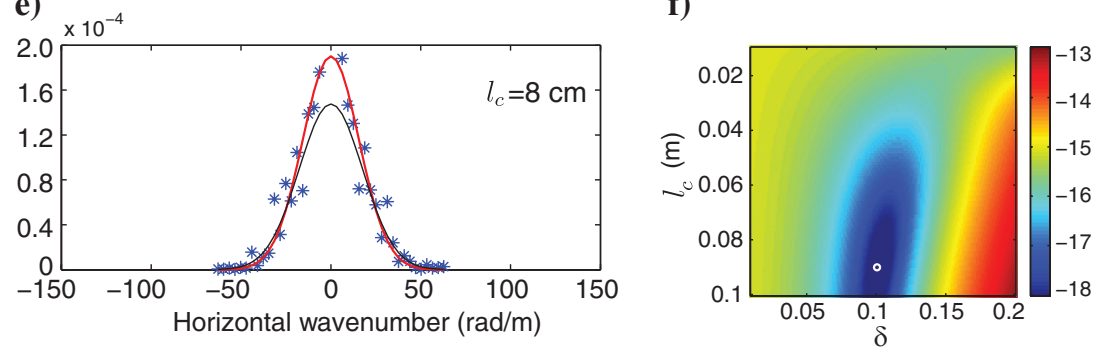

Figure 7. (a) PSD of stress field at the fracture depth, for a sparse receiver spacing $\Delta x=0.05 \mathrm{~m}$, estimated using the heterogeneous compliance model with $l_{c}=4 \mathrm{~cm}$ (blue asterisks). Red line and black line show the predicted results from grid search optimization and the true value calculated by perturbation theory (equation 16), respectively. (b) Energy function for grid search in the $\log$ scale $\left(\log _{10} E\left(\delta, l_{c}\right)\right)$ for (a). (c and d) are similar to (a and b) but using the model with $l_{c}=2 \mathrm{~cm}$. (e and f) are similar to (a and $\mathrm{b}$ ) but using the model with $l_{c}=8 \mathrm{~cm}$.

Table 2. Estimated $\delta$ and $l_{c}$ by least-square optimization for the heterogeneous model of the fracture with $l_{c}=2 \mathrm{~cm}$.

\begin{tabular}{lcccccc}
$\Delta x(\mathrm{~m})$ & $z_{0}^{\text {est }}$ & $\eta_{N}^{0(\text { est })}$ & $V_{\mathrm{P}}^{\text {est }}$ & $\mathrm{S} / \mathrm{N}$ & $\delta$ & $l_{c}(\mathrm{~m})$ \\
\hline True model & $z_{0}$ & $\eta_{N}^{0}$ & $V_{\mathrm{P}}$ & $\infty$ & 0.100 & 0.020 \\
0.05 & $z_{0}$ & $\eta_{N}^{0}$ & $V_{\mathrm{P}}$ & $\infty$ & $0.138 \pm 0.001$ & $0.012 \pm 0.001$ \\
\hline
\end{tabular}


by least-square optimization when a constant amplitude has been subtracted from the data. The amplitude of this offset is set to wavenumber components in the range $k_{x}=200-300$. This correction may not be appropriate for the components around the incident wavenumber $\left(k_{x}^{\mathrm{inc}}=0\right)$ because they contain the interaction between the signal and the noise. However, it is clear that the PSD of the stress field (red line in Figure 9) and the corresponding parameters $\left(\delta=0.097 \pm 0.001, l_{c}=0.037 \pm 0.001 \mathrm{~m}\right)$ are much better estimated after the correction. $2.99 \times 10^{-5}$, which is the average value of data amplitude at higher

\section{CONCLUSION}

Prior to this work, there was no approach to estimate from the scattered elastic wavefield the statistical property of the heterogeneous compliance distribution. In this paper, we have proposed a new method to estimate the PSD of the spatially heterogeneous distribution of fracture compliance using the scattered wavefield.

We have applied perturbation theory to derive the relation between the scattered elastic waves and the PSD of the spatially heterogeneous compliance. PSD of the compliance has been derived from the stress field estimated at the fracture depth, followed by a least-square optimization of the predicted PSD of the stress field. This has been achieved by analyzing the first-order solution of the scattered wavefield for an incident plane wave.

We have tested the approach numerically. For this purpose, we have considered a 2D geometry and incident plane waves. Our results show that the estimation of the standard deviation $\delta$ and the correlation length $l_{c}$ of the fracture compliance, assuming a Gaussian PSD of the compliance, is Table 3. Estimated $\delta$ and $l_{c}$ by least-square optimization for the heterogeneous model of the fracture with $l_{c}=8 \mathrm{~cm}$.

\begin{tabular}{lcccccc}
$\Delta x(\mathrm{~m})$ & $z_{0}^{\text {est }}$ & $\eta_{N}^{0(\text { est })}$ & $V_{P}^{\text {est }}$ & $\mathrm{S} / \mathrm{N}$ & $\delta$ & $l_{c}(\mathrm{~m})$ \\
\hline True model & $z_{0}$ & $\eta_{N}^{0}$ & $V_{\mathrm{P}}$ & $\infty$ & 0.100 & 0.080 \\
0.05 & $z_{0}$ & $\eta_{N}^{0}$ & $V_{\mathrm{P}}$ & $\infty$ & $0.107 \pm 0.001$ & $0.090 \pm 0.001$ \\
\hline
\end{tabular}

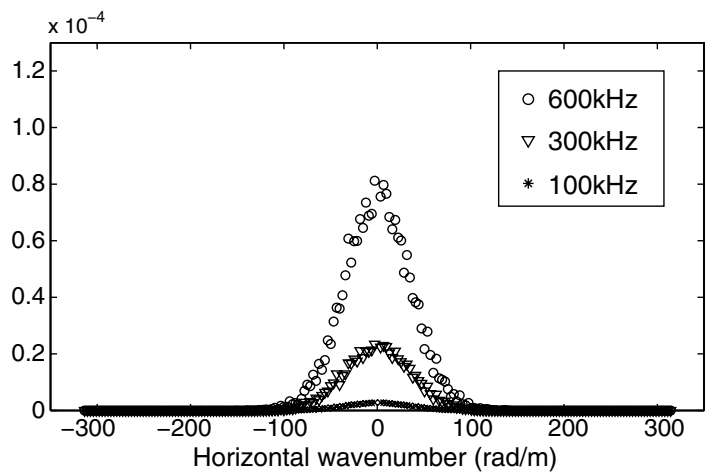

Figure 8. PSD of stress field at the fracture depth calculated using wdSDD for three different incident wave frequencies $(100 \mathrm{kHz}$, $300 \mathrm{kHz}, 600 \mathrm{kHz}$ ).

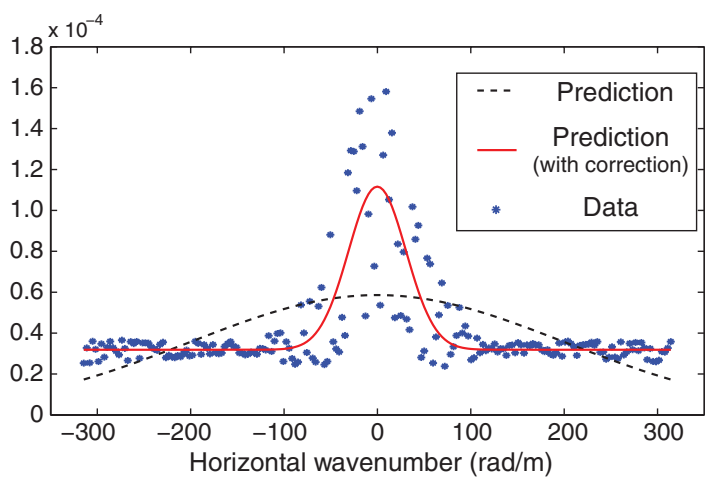

Figure 9. The prediction of PSD of the stress field using data contaminated with white noise with $\mathrm{S} / \mathrm{N}=150$ (blue line). The black dashed line shows the prediction by grid search optimization of the energy function. The red line illustrates the improved prediction after correcting for the amplitude shift due to the noise. quite robust against errors in input fracture depth. Errors in the values of other input parameters (viz. propagation velocity and background compliance) affect the estimation of $\delta$ more than that of $l_{c}$. The estimates are also robust against sparse sampling of the wavefield, as long as the sparse data contain the wavenumber components which give the characteristics of the slope of the PSD of the stress field. When the sampling intervals are large compared to the correlation length of the heterogeneous compliance distribution, it is difficult to estimate the accurate PSD of the compliance due to the spatial aliasing and the small amplitude variation within the available wavenumbers. The use of higher frequencies should offer a better PSD estimate because the higher frequencies cause a stronger amplitude variation and therefore a higher sensitivity in the least-square optimization. Further, we have investigated the effect of white noise in the PSD estimation. The contaminated data shows larger amplitudes; a change in the shape of the PSD of the stress field significantly affects the PSD estimation, especially for small $\mathrm{S} / \mathrm{N}$. In this case, an amplitude correction should lead to a much better estimate of $\delta$ and $l_{c}$.

\section{ACKNOWLEDGMENTS}

This work is supported by The Netherlands Research Centre for Integrated Solid Earth Science.

\section{APPENDIX A \\ FORWARD AND INVERSE WAVEFIELD EXTRAPOLATION OPERATOR}

In the derivation of an exact solution for the scattering response and perturbation theory (equations 8 and 9), the incident wavefield and the scattered wavefield are considered to be at the fracture plane in the subsurface. Therefore, we need to propagate the wavefield from the receiver depth to the fracture depth, or vice versa. We achieve this by applying a forward wavefield extrapolation operator (e.g., Wapenaar and Berkhout, 1989; Wapenaar and Haime, 1990) 
to the potential wave vector $\mathbf{D}^{ \pm}$. In the $f-k$ domain, the downgoing wavefield at the fracture depth $z_{0}$ is obtained by extrapolating the downgoing wavefield at the receiver $z_{r}$

$$
\hat{\mathbf{D}}^{+}\left(z_{0}\right)=\hat{\mathbf{W}}^{+}\left(z_{0}, z_{r}\right) \hat{\mathbf{D}}^{+}\left(z_{r}\right)
$$

where $z_{r}<z_{0}$ and $\hat{\mathbf{W}}^{+}\left(z_{0}, z_{r}\right)$ is the downgoing forward wavefield extrapolation operator. Similarly, the upgoing wavefield at $z_{r}$ is obtained by extrapolation of the upgoing wavefield at $z_{0}$

$$
\hat{\mathbf{D}}^{-}\left(z_{r}\right)=\hat{\mathbf{W}}^{-}\left(z_{r}, z_{0}\right) \hat{\mathbf{D}}^{-}\left(z_{0}\right),
$$

where $\hat{\mathbf{W}}^{-}\left(z_{r}, z_{0}\right)$ is the upgoing forward wavefield extrapolation operator. In case of isotropic homogeneous media, these operators read

$$
\hat{\mathbf{W}}^{+}\left(z_{0}, z_{r}\right)=\hat{\mathbf{W}}^{-}\left(z_{r}, z_{0}\right)=\left(\begin{array}{ccc}
e^{-i k_{z}^{P} \Delta z} & 0 & 0 \\
0 & e^{-i k_{z}^{S} \Delta z} & 0 \\
0 & 0 & e^{-i k_{z}^{S} \Delta z}
\end{array}\right)
$$

where,

$$
\begin{aligned}
\Delta z= & \left|z_{0}-z_{r}\right|, \\
k_{z}^{P}= & \left(\omega^{2} / V_{P}^{2}-k_{r}^{2}\right)^{1 / 2}, \\
k_{z}^{S}= & \left(\omega^{2} / V_{S}^{2}-k_{r}^{2}\right)^{1 / 2}, \\
& \mathfrak{\Im}\left[k_{z}^{P}\right], \mathfrak{\Im}\left[k_{z}^{S}\right] \leq 0, \\
k_{r}^{2}= & k_{x}^{2}+k_{y}^{2} .
\end{aligned}
$$

Here, $\mathfrak{I}$ denotes the imaginary component. By using $\hat{\mathbf{W}}^{ \pm}$, one can calculate the incident field at the fracture depth and the scattered response at the receiver depth. When the horizontal wavenumber $k_{r}$ is larger than its values at the turning-points $\left(\omega / V_{P}\right.$ or $\left.\omega / V_{S}\right)$, the vertical wavenumbers $\left(k_{z}^{P}\right.$ or $\left.k_{z}^{S}\right)$ become purely imaginary. This corresponds to the horizontally propagating waves whose amplitude decays exponentially with the distance from the fracture (viz. evanescent waves).

For the estimation of PSD of the heterogeneous compliance, we need to estimate the stress field at the fracture depth from the scattered response at the receiver depth. We achieve this by using the inverse wavefield extrapolation operator. In the $f-k$ domain, this is the inverse matrix of the forward wavefield extrapolation operator (equation A-3). However, to suppress the instability arising due to the evanescent waves, we have adopted a matched inverse operator (e.g., Wapenaar and Berkhout, 1989; Wapenaar et al., 1989) as an approximate inverse wavefield extrapolation operator. Therefore, the upgoing wave vector at the fracture depth is estimated using the upgoing wave vector at the receiver depth

$$
\hat{\mathbf{D}}^{-}\left(z_{0}\right)=\hat{\mathbf{F}}^{-}\left(z_{0}, z_{r}\right) \hat{\mathbf{D}}^{-}\left(z_{r}\right),
$$

where $\hat{\mathbf{F}}^{-}\left(z_{0}, z_{r}\right)$ is the matched inverse operator

$$
\hat{\mathbf{F}}^{-}\left(z_{0}, z_{r}\right)=\left(\hat{\mathbf{W}}^{-}\left(z_{r}, z_{0}\right)\right)^{\dagger} .
$$

Equation A-6 indicates that the matched operator is an exact inverse extrapolation operator for the propagating wave $\left(k_{r}^{2} \leq \omega^{2} / V_{P}^{2}\right.$ and $\left.k_{r}^{2} \leq \omega^{2} / V_{S}^{2}\right)$. This operator exponentially suppresses the amplitude of the evanescent waves $\left(k_{r}^{2}>\omega^{2} / V_{\mathrm{P}}^{2}\right.$ and $\left.k_{r}^{2}>\omega^{2} / V_{\mathrm{S}}^{2}\right)$.

Note that, in the region of the propagating waves, the forward and the inverse wavefield operators act as phase-shift operators which contain the propagation velocity of the medium and the distance of propagation $(\Delta z)$.

\section{APPENDIX B}

\section{APPLICATION OF PERTURBATION THEORY TO THE TRANSMISSION AND REFLECTION PROBLEM}

We derive the approximate solution for the transmission/ reflection problem (equations $8-10$ ) by using perturbation theory. We assume the relation $\mathbf{Z}(x, y)=\mathbf{Z}_{0}+\epsilon \mathbf{Z}_{1}(x, y)$ (equation 7). We also assume that the potential wavefields $\left(\hat{\mathbf{D}}_{l}^{+}\right.$and $\left.\hat{\mathbf{D}}_{u}^{-}\right)$and the stress field $\left(\hat{\boldsymbol{\tau}}_{u}\right)$ in equation 3 can be expanded into power series of $\epsilon$ as

$$
\begin{array}{r}
\hat{\mathbf{D}}_{l}^{+}=\hat{\mathbf{D}}_{l}^{+(0)}+\epsilon \hat{\mathbf{D}}_{l}^{+(1)}+\epsilon^{2} \hat{\mathbf{D}}_{l}^{+(2)}+\ldots, \\
\hat{\mathbf{D}}_{u}^{-}=\hat{\mathbf{D}}_{u}^{-(0)}+\epsilon \hat{\mathbf{D}}_{u}^{-(1)}+\epsilon^{2} \hat{\mathbf{D}}_{u}^{-(2)}+\ldots, \\
\hat{\boldsymbol{\tau}}_{u}=\hat{\boldsymbol{\tau}}_{u}^{(0)}+\epsilon \hat{\boldsymbol{\tau}}_{u}^{(1)}+\epsilon^{2} \hat{\boldsymbol{\tau}}_{u}^{(2)}+\ldots
\end{array}
$$

Fourier-transforming equation 7 and substituting these equations into equation 3 yields

$$
\begin{aligned}
& \hat{\mathbf{L}}_{l}\left(\begin{array}{c}
\hat{\mathbf{D}}_{l}^{+(0)} \\
\mathbf{0}
\end{array}\right)+\epsilon \hat{\mathbf{L}}_{l}\left(\begin{array}{c}
\hat{\mathbf{D}}_{l}^{+(1)} \\
\mathbf{0}
\end{array}\right)+\ldots \\
& =\hat{\mathbf{L}}_{u}\left(\begin{array}{c}
\hat{\mathbf{D}}^{\mathrm{inc}} \\
\hat{\mathbf{D}}_{u}^{-(0)}
\end{array}\right)+\left(\begin{array}{c}
i \omega \mathbf{Z}_{0} \hat{\boldsymbol{\tau}}_{u}^{(0)} \\
\mathbf{0}
\end{array}\right) \\
& +\epsilon\left[\hat{\mathbf{L}}_{u}\left(\begin{array}{c}
\mathbf{0} \\
\hat{\mathbf{D}}_{u}^{-(1)}
\end{array}\right)+\left(\begin{array}{c}
i \omega \mathbf{Z}_{0} \hat{\boldsymbol{\tau}}_{u}^{(1)}+i \omega \hat{\mathbf{Z}}_{1} * \hat{\boldsymbol{\tau}}_{u}^{(0)} \\
\mathbf{0}
\end{array}\right)\right] \\
& +\ldots
\end{aligned}
$$

We can simplify equation B-4 to the zeroth order of $\epsilon$ (i.e., $\epsilon^{0}$ ) and reach an approximate solution for the transmission and reflection problem:

$$
\begin{aligned}
\hat{\mathbf{L}}_{l}\left(\begin{array}{c}
\hat{\mathbf{D}}_{l}^{+(0)} \\
\mathbf{0}
\end{array}\right) & =\hat{\mathbf{L}}_{u}\left(\begin{array}{c}
\hat{\mathbf{D}}^{\mathrm{inc}} \\
\hat{\mathbf{D}}_{u}^{-(0)}
\end{array}\right)+\left(\begin{array}{c}
i \omega \mathbf{Z}_{0} \hat{\boldsymbol{\tau}}_{u}^{(0)} \\
\mathbf{0}
\end{array}\right) \\
& =\left(\begin{array}{cc}
\mathbf{I} & i \omega \mathbf{Z}_{0} \\
\mathbf{0} & \mathbf{I}
\end{array}\right) \hat{\mathbf{L}}_{u}\left(\begin{array}{c}
\hat{\mathbf{D}}^{\mathrm{inc}} \\
\hat{\mathbf{D}}_{u}^{-(0)}
\end{array}\right) .
\end{aligned}
$$

Similarly, we obtain the relation for the first order of $\epsilon$ (i.e., $\epsilon^{1}$ ) as 


$$
\begin{gathered}
\hat{\mathbf{L}}_{l}\left(\begin{array}{c}
\hat{\mathbf{D}}_{l}^{+(1)} \\
\mathbf{0}
\end{array}\right)=\hat{\mathbf{L}}_{u}\left(\begin{array}{c}
\mathbf{0} \\
\hat{\mathbf{D}}_{u}^{-(1)}
\end{array}\right)+\left(\begin{array}{cc}
i \omega \mathbf{Z}_{0} \hat{\boldsymbol{\tau}}_{u}^{(1)}+i \omega \hat{\mathbf{Z}}_{1} * \hat{\boldsymbol{\tau}}_{u}^{(0)} \\
\mathbf{0}
\end{array}\right) \\
=\left(\begin{array}{cc}
\mathbf{I} & i \omega \mathbf{Z}_{0} \\
\mathbf{0} & \mathbf{I}
\end{array}\right) \hat{\mathbf{L}}_{u}\left(\begin{array}{c}
\mathbf{0} \\
\hat{\mathbf{D}}_{u}^{-(1)}
\end{array}\right)+\left(\begin{array}{c}
i \omega \hat{\mathbf{Z}}_{1} * \hat{\boldsymbol{\tau}}_{u}^{(0)} \\
\mathbf{0}
\end{array}\right) .
\end{gathered}
$$

For the $n$th order of $\epsilon$ (i.e., $\epsilon^{n}, n>0$ ), this can be generalized as in equations 9 and 10 .

\section{APPENDIX C \\ DERIVATION OF PSD OF THE PERTURBED COMPLIANCE IN THE FIRST-ORDER SOLUTION}

Here, we derive equation 15 . In isotropic homogeneous media, the explicit form of $\hat{\mathbf{H}}$, as given by Nakagawa et al. (2004), is

$$
\hat{\mathbf{H}}=\frac{2}{\rho V_{S} R} \mathbf{R}^{T}\left(\begin{array}{ccc}
k_{z}^{S} / k_{S} & 0 & 0 \\
0 & k_{S} R / k_{z}^{S} & 0 \\
0 & 0 & k_{z}^{P} / k_{S}
\end{array}\right) \mathbf{R},
$$

where $\mathbf{R}$ and $R$ are the rotation matrix and the Rayleigh function, respectively

$$
\begin{gathered}
\mathbf{R}=\left(\begin{array}{ccc}
k_{x} / k_{r} & k_{y} / k_{r} & 0 \\
-k_{y} / k_{r} & k_{x} / k_{r} & 0 \\
0 & 0 & 1
\end{array}\right), \\
R=\left(1-2\left(k_{r} / k_{S}\right)^{2}\right)^{2}+4\left(k_{r} / k_{S}\right)^{2}\left(k_{z}^{P} k_{z}^{S} / k_{S}^{2}\right) .
\end{gathered}
$$

Note that the rotation matrix has the property $\mathbf{R R}^{T}=\mathbf{I}$. Substituting equation $\mathrm{C}-1$ into equation 12 yields

$$
\hat{\boldsymbol{\tau}}_{u}^{-(1)}\left(\mathbf{k} ; \mathbf{k}^{\mathrm{inc}}\right)=(i q / 2)^{2} R R^{\mathrm{inc}} \mathbf{R}^{T} \mathbf{U R}\left(\mathbf{R}^{\mathrm{inc}}\right)^{T} \mathbf{U}^{\mathrm{inc}} \mathbf{R}^{\mathrm{inc}} \hat{\boldsymbol{\tau}}^{c},
$$

where we define the term $q=\rho V_{\mathrm{S}} \omega \eta_{N}^{0}$ and $\mathbf{R}^{\text {inc }}$ and $R^{\text {inc }}$ are the rotation matrix and the Rayleigh function in the incident wavenumber, i.e., $\mathbf{R}^{\text {inc }}=\mathbf{R}\left(\mathbf{k}^{\text {inc }}\right)$ and $R^{\text {inc }}=R\left(\mathbf{k}^{\text {inc }}\right)$. The matrix $\mathbf{U}$ and $\mathbf{U}^{\text {inc }}$ are the diagonal matrices

$$
\mathbf{U}=\left(\begin{array}{ccc}
u_{1} & 0 & 0 \\
0 & u_{2} & 0 \\
0 & 0 & u_{3}
\end{array}\right), \quad \mathbf{U}^{\text {inc }}=\left(\begin{array}{ccc}
u_{1}^{\text {inc }} & 0 & 0 \\
0 & u_{2}^{\text {inc }} & 0 \\
0 & 0 & u_{3}^{\text {inc }}
\end{array}\right)
$$

where the indices of these matrices are

$$
\begin{aligned}
u_{1} & =\frac{\nu}{k_{z}^{S} / k_{S}-i q R \nu / 2} \frac{\eta_{T}^{1}\left(\mathbf{k}-\mathbf{k}^{\mathrm{inc}}\right)}{\eta_{T}^{0}}, \\
u_{2} & =\frac{\nu}{k_{S} R / k_{z}^{S}-i q R \nu / 2} \frac{\eta_{T}^{1}\left(\mathbf{k}-\mathbf{k}^{\mathrm{inc}}\right)}{\eta_{T}^{0}}, \\
u_{3} & =\frac{1}{k_{z}^{P} / k_{S}-i q R / 2} \frac{\eta_{N}^{1}\left(\mathbf{k}-\mathbf{k}^{\mathrm{inc}}\right)}{\eta_{N}^{0}}, \\
u_{1}^{\text {inc }} & =\frac{2}{i q R^{\text {inc }}}+\frac{\nu}{k_{z}^{S}\left(\mathbf{k}^{\text {inc }}\right) / k_{S}-i q R^{\text {inc }} \nu / 2}, \\
u_{2}^{\text {inc }} & =\frac{2}{i q R^{\text {inc }}}+\frac{\nu}{k_{S} R^{\text {inc }} / k_{z}^{S}\left(\mathbf{k}^{\text {inc }}\right)-i q R^{\text {inc }} \nu / 2}, \\
u_{3}^{\text {inc }} & =\frac{2}{i q R^{\text {inc }}}+\frac{1}{k_{z}^{P}\left(\mathbf{k}^{\text {inc }}\right) / k_{S}-i q R^{\text {inc }} / 2} .
\end{aligned}
$$

Here, we assume $\eta_{T}^{0}=\nu \eta_{N}^{0}$. Note that the variables with the subscript "inc" have constant values for all wavenumber $\mathbf{k}$ with a known incident wavenumber $\mathbf{k}^{\text {inc }}$. Due to the property of the rotation matrix $\mathbf{R}$ and the diagonal matrix $\mathbf{U}$, calculating the power of the stress field $\left(\epsilon^{2}\left(\hat{\boldsymbol{\tau}}_{u}^{-(1)}\right)^{\dagger} \hat{\boldsymbol{\tau}}_{u}^{-(1)}\right)$ we get the summation of $S_{T}\left(\mathbf{k}-\mathbf{k}^{\text {inc }}\right)$ and $S_{N}\left(\mathbf{k}-\mathbf{k}^{\text {inc }}\right)$ together with their respective coefficients. Here, $S_{T}$ and $S_{N}$ are the scaled power spectra of the perturbed compliance distributions defined as

$$
S_{T}(\mathbf{k})=\left|\epsilon \eta_{T}^{1}(\mathbf{k}) / \eta_{T}^{0}\right|^{2}, \quad S_{N}(\mathbf{k})=\left|\epsilon \eta_{N}^{1}(\mathbf{k}) / \eta_{N}^{0}\right|^{2} .
$$

From the definition of PSD, taking the ensemble average and the limit to the power of the stress field, we obtain equation 15 , with the PSD of the perturbed compliance defined as

$$
P_{T}(\mathbf{k})=\lim _{A \rightarrow \infty} E\left\langle\frac{S_{T}(\mathbf{k})}{A}\right\rangle, \quad P_{N}(\mathbf{k})=\lim _{A \rightarrow \infty} E\left\langle\frac{S_{N}(\mathbf{k})}{A}\right\rangle .
$$

To derive equations C-8 and 15, we assume the functions $\hat{\boldsymbol{\tau}}_{u}^{-}(\mathbf{k})$, $\eta_{T}^{1}(\mathbf{k})$ and $\eta_{N}^{1}(\mathbf{k})$ to be the Fourier transform of the truncated version of the random process (e.g., Miller and Childers, 2012). Note that we assume $\eta_{T}^{1}=\nu \eta_{N}^{1}$ to derive equation 15 , which contains $P_{N}$ instead of a summation of $P_{N}$ and $P_{T}$. The coefficient $f\left(\mathbf{k} ; \omega, \mathbf{k}^{\text {inc }}, V_{\mathrm{S}}, V_{\mathrm{P}}, \rho, \hat{\boldsymbol{\tau}}^{c}, \nu\right)$ in equation 15 can be calculated using equations C-4-C-6. Equations C-7 and C-8 state that the PSD of the perturbed compliance is defined to be scaled by the background compliance. Therefore, the value of $\delta$ in the Gaussian PSD (equation 19) is considered as a value relative to the background compliance. For example, $\delta=0.1$ indicates that the standard deviation of the perturbed compliance is $10 \%$ of the background value. From this discussion, we use the value of $\delta$ for the perturbation magnitude $\epsilon$ in equation 7 .

\section{REFERENCES}

Brown, S., 1995, Simple mathematical model of a rough fracture: Journal of Geophysical Research, 100, 5941-5952, doi: 10.1029/94JB03262.

Brown, S., and C. Scholz, 1985, Closure of random elastic surfaces in contact: Journal of Geophysical Research, 90, 5531-5545, doi: 10.1029/ JB090iB07p05531.

Haugen, G., and M. Schoenberg, 2000, The echo of a fault or fracture: Geophysics, 65, 176-189, doi: 10.1190/1.1444708.

Hudson, J. A., E. Liu, and S. Crampin, 1996, Transmission properties of a plane fault: Geophysical Journal International, 125, 559-566, doi: 10 .1111/j.1365-246X.1996.tb00018.x. 
Kay, S., and S. Marple, 1981, Spectrum analysis-A modern perspective: Proceedings of the IEEE, 69, 1380-1419, doi: 10.1109/PROC.1981 .12184 .

Kuperman, W. A., 1975, Coherent component of specular reflection and transmission at a randomly rough 2-fluid interface: Journal of the Acoustical Society of America, 58, 365-370, doi: 10.1121/1.380680.

Lanaro, F., 2000, A random field model for surface roughness and aperture of rock fractures: International Journal of Rock Mechanics and Mining Sciences and Geomechanics Abstracts, 37, 1195-1210, doi: 10.1016/ S1365-1609(00)00052-6.

Leary, P. C., 1995, Quantifying crustal fracture heterogeneity by seismic scattering: Geophysical Journal International, 122, 125-142, doi: 10 $.1111 /$ j.1365-246X.1995.tb03541.x.

Leiderman, R., P. E. Barbone, and A. M. B. Braga, 2007, Reconstructing the adhesion stiffness distribution in a laminated elastic plate: Exact and approximate inverse scattering solutions: Journal of the Acoustical Society of America, 122, 1906-1916, doi: 10.1121/1.2772212.

Leiderman, R., A. M. B. Braga, and P. E. Barbone, 2005, Scattering of ultrasonic waves by defective adhesion interfaces in submerged laminated plates: Journal of the Acoustical Society of America, 118, 2154-2166, doi: $10.1121 / 1.2036147$

Lubbe, R., J. Sothcott, M. Worthington, and C. McCann, 2008, Laboratory estimates of normal and shear fracture compliance: Geophysical Prospecting, 56, 239-247, doi: 10.1111/j.1365-2478.2007.00688.x.

Miller, S. L., and D. Childers, 2012, Probability and random processes: With applications to signal processing and communications: Elsevier Academic Press.

Minato, S., and R. Ghose, 2013, Inverse scattering solution for the spatially heterogeneous compliance of a single fracture: Geophysical Journa International, 195, 1878-1891, doi: 10.1093/gji/ggt348.

Nakagawa, S., K. T. Nihei, and L. R. Myer, 2004, Plane wave solution for elastic wave scattering by a heterogeneous fracture: Journal of the Acoustical Society of America, 115, 2761-2772, doi: 10.1121/1.1739483.

Pardo-Iguzquiza, E., and M. Chica-Olmo, 1993, The fourier integral method: An efficient spectral method for simulation of random fields: Mathematical Geology, 25, 177-217, doi: 10.1007/BF00893272.

Pyrak-Nolte, L., and J. Morris, 2000, Single fractures under normal stress: The relation between fracture specific stiffness and fluid flow: International Journal of Rock Mechanics and Mining Sciences and Geomechanics Abstracts, 37, 245-262, doi: 10.1016/S1365-1609(99) 00104-5.

Pyrak-Nolte, L., L. Myer, and N. Cook, 1990, Transmission of seismic waves across single natural fractures: Journal of Geophysical Research, 95, 8617-8638, doi: 10.1029/JB095iB06p08617.
Pyrak-Nolte, L., and D. Nolte, 1992, Frequency dependence of fracture stiffness: Geophysical Research Letters, 19, 325-328, doi: 10.1029/ 91GL03179.

Sayers, C. M., 2002, Fluid-dependent shear-wave splitting in fractured media: Geophysical Prospecting, 50, 393-401, doi: 10.1046/j.1365-2478 $.2002 .00324 . x$.

Scherbaum, F., and H. Sato, 1991, Inversion of full seismogram envelopes based on the parabolic approximation: Estimation of randomness and attenuation in southeast Honshu, Japan: Journal of Geophysical Research, 96, 2223-2232, doi: 10.1029/90JB01538.

Schoenberg, M., 1980, Elastic wave behavior across linear slip interfaces: Journal of the Acoustical Society of America, 68, 1516-1521, doi: 10 $.1121 / 1.385077$

Shapiro, S. A., and P. Hubral, 1999, Elastic waves in random media: Lecture notes in earth sciences: Berlin Springer Verlag, 80.

Thorsos, E. I., and D. R. Jackson, 1989, The validity of the perturbation approximation for rough surface scattering using a Gaussian roughness spectrum: Journal of the Acoustical Society of America, 86, 261-277, doi: $10.1121 / 1.398342$.

Toomey, A., C. J. Bean, and O. Scotti, 2002, Fracture properties from seismic data - A numerical investigation: Geophysical Research Letters, 29, 9-1-9-4, doi: 10.1029/2001GL013867.

Ursin, B., 1983, Review of elastic and electromagnetic wave propagation in horizontally layered media: Geophysics, 48, 1063-1081, doi: 10.1190/1 .1441529 .

van Der Neut, J., M. Sen, and K. Wapenaar, 2008, Seismic reflection coefficients of faults at low frequencies: A model study: Geophysical Prospecting, 56, 287-292, doi: 10.1111/j.1365-2478.2008.00701.x.

Wapenaar, C., and A. Berkhout, 1989, Elastic wave field extrapolation: Redatuming of single-and multi-component seismic data: Elsevier Science.

Wapenaar, C., G. Peels, V. Budejicky, and A. Berkhout, 1989, Inverse extrapolation of primary seismic waves: Geophysics, 54, 853-863, doi: 10.1190/1.1442714.

Wapenaar, C. P. A., and G. C. Haime, 1990, Elastic extrapolation of primary seismic P- and S-waves: Geophysical Prospecting, 38, 23-60, doi: 10 $.1111 / \mathrm{j} .1365-2478.1990 . \mathrm{tb01833}$.x.

Willis, M., D. Burns, R. Rao, B. Minsley, M. Toksoz, and L. Vetri, 2006 Spatial orientation and distribution of reservoir fractures from scattered seismic energy: Geophysics, 71, no. 5, O43-O51, doi: 10.1190/1 .2235977 .

Woodhouse, J. H., 1974, Surface waves in a laterally varying layered structure: Geophysical Journal of the Royal Astronomical Society, 37, 461490, doi: 10.1111/j.1365-246X.1974.tb04098.x. 